\title{
4 Korpus: Theaterproben in audiovisuellen und ethnographischen Daten
}

Wie alle sozialen Situationen werden auch Theaterproben von den Teilnehmenden in der Interaktion hergestellt. Mit Garfinkels (1967) Ethnomethodologie gedacht, erzeugt die Gesamtheit der Aktivitäten aller Beteiligten gemeinsam die Situation Theaterprobe. Diese hergestellte Situation wiederum wirkt reflexiv auf die Beteiligten und beeinflusst die Möglichkeiten der interaktionalen Ausgestaltung der sozialen Situation. Theaterproben sind demnach zu verstehen als Aktivitätskomplexe innerhalb der Institution Theater mit dem spezifischen Ziel der Erarbeitung eines Theaterstücks innerhalb von ca. sechs Wochen ${ }^{20}$ zum Zweck der Aufführung vor Publikum. Aus dieser Definition ergeben sich zwei zentrale Merkmale, die bei der Betrachtung von kommunikativen Prozessen innerhalb von Theaterproben berücksichtigt werden müssen: die institutionelle Rahmung und die Zielorientierung des gemeinsamen Projekts. Um die Verfahren der Koordinierung multipler Aktivitäten in Theaterproben nachvollziehen zu können, bedarf es daher einer ethnographischen Beschreibung der Institution, der (audio-visuellen) Datenerhebung sowie einer Reflexion der teilnehmenden Beobachtung im Feld.

\subsection{Theaterproben als Forschungsgegenstand}

Theaterproben sind natürliche Laboratorien (Krug et al. 2020), in denen Beteiligte in einem räumlich isolierten und durch Probenzeiten temporal begrenzten Umfeld schrittweise an den Formen einer Inszenierung arbeiten. In Theaterproben werden damit die gleichen Handlungen von denselben Interagierenden solange wiederholt, bis deren gefestigter Zustand ausgehandelt ist. Dies umfasst die Erarbeitung, das Finden und die Prüfung der Szenen durch die Beteiligten. Dadurch entstehen durch die Teilnehmenden selbst organisierte Fallkollektionen ähnlicher kommunikativer Prozesse. Obwohl dies eine ideale Voraussetzung für konversantionsanalytische Arbeiten darstellt, wurden Theaterproben sowohl in der Konversationsanalyse als auch in der Theaterwissenschaft bislang nur wenig beforscht. So stellt Matzke (2012: 29) für die Theaterwissenschaft fest:

20 Roselt (2011: 21) schreibt zur Dauer des Probenprozesses in modernen Theatern: „Sechs Wochen Proben sind für eine Neuinszenierung gegenwärtig keine Ausnahme.“ 
In der theaterwissenschaftlichen Forschung gibt es bisher keine systematischen Untersuchungen zur Probenpraxis, zu Formen der Organisation theatraler Arbeit, ebenso wenig zum Zusammenhang von Theaterstruktur im Sinne einer Arbeitsorganisation und theatraler Ästhetik. Auch eine grundlegende Untersuchung zur Geschichte des Probens fehlt. [...] Die Probe entzieht sich bisher einer theoretischen Bestimmung.

Wenn über Proben im Rahmen von Inszenierungsanalysen gesprochen wird, dann meist auf Grundlange anekdotischer Evidenz (Düffel 2011; Eikels 2011) oder mithilfe ethnographischer Studien (McAuley 2012; Wartemann 2011). Dabei „reduziert sich [die Auseinandersetzung mit der Probe] oft auf eine Reflexion schauspieltheoretischer Fragen“ (Matzke 2012: 103). Bei der dadurch entstehenden Zentrierung auf Regieführende und Schauspieler`innen finden die Beiträge anderer bei der Probe anwesender Personen kaum Beachtung (Stegemann 2011). An dieser Stelle kann die konversationsanalytische Methodologie helfen, Proben als interaktionale Hervorbringung aller an ihnen Beteiligten zu verstehen. Jedoch wird auch konversationsanalytisch bislang nur selten die kommunikative Arbeit während einer Theaterprobe fokussiert und stattdessen die Handlungen der Figuren auf der Bühne rekonstruiert:

Where there has been some application of the CA methods to dramatic texts, it has focused almost exclusively on the play scripts [...], rather than the processes of enacting representations of social interaction.

(Hazel 2015: 47)

Dafür könnte es zwei entscheidende Gründe geben. Zum ersten ist der Feldzugang zu professionellen Theatern schwierig, weil Theaterschaffende nur selten Externe in ihrem „Schutzraum“ (Matzke 2012: 88) dulden. Zum zweiten ist bei Analysen oft umfangreiches ethnographisches Wissen Voraussetzung dafür, die Handlungen auf der Probebühne überhaupt verstehen zu können (z. B. in Bezug auf Fachsprache oder geteiltes Wissen bzgl. einzelner Prozesse). Daher ist es nicht verwunderlich, dass die meisten Forschungsprojekte zu Theaterinteraktionen die kommunikativen Prozesse zwischen Theaterschaffenden und ihrem Publikum auf der Bühne und im Anschluss im Theaterfoyer fokussieren (Broth 2011; Fix 2017; Habscheid 2015; Hrncal \& Gerwinski 2015). Aktuell gibt es daher nur wenige empirische Studien zur Probenkommunikation. Diese untersuchen unter anderem longitudinale Prozesse des Probens (Hazel 2015, 2018), analysieren die Organisation der Übergänge zwischen Spiel- und Realwelt (Schmidt 2014), rekonstruieren Handlungsausrichtungen an zukünftigen Ereignissen (Schmidt 2017, 2018), fokussieren die kollaborativen Prozesse (Milde 2019) oder diskutieren Regieanweisungen als zentrale Probenhandlungen (Lefebvre 2018; Krug 2020b; Wessel 2020). All diese Studien haben neben ihrer interaktionsanalytischen Ausrichtung gemeinsam, dass sie auf teilweise immensen Videokorpora basieren und dadurch die multimodale Perspektive in ihre Analysen integrieren können. Darüber hinaus 
bearbeiten sie Fragestellungen, die dezidiert im Setting Theaterprobe verortet sind. Die vorliegende Studie hat hingegen das Ziel, die koordinativen Verfahren bei der Bearbeitung simultan relevanter Handlungssequenzen herauszustellen. Das Setting dient hier lediglich als natürliches Laboratorium, in dem das Auftreten multipler Aktivitäten systematisch zu beobachten ist. Daher bilden neben Situationen des szenischen Probierens ${ }^{21}$ auch Pausengespräche, Konzeptdiskussionen und Interaktionen vor bzw. nach der eigentlichen Probe die Datengrundlage für die vorliegende Untersuchung.

\subsection{Daten: 31 Proben einer Stückentwicklung}

Wie bei vielen ethnographischen Arbeiten stellt der Feldzugang zu den Proben eines professionellen Theaterhauses eine der größten Hürden einer ethnographischen Datenerhebung dar. Um das dieser Untersuchung zugrundeliegende Korpus erheben zu können, bot ich einem bekannten, professionellen Regisseur $^{22}$ an, als Videokünstler die im Stück verwendeten Bewegtbildinstallationen zu erstellen. Im Gegenzug durfte ich alle 31 Proben seines aktuellen Theaterprojekts mit mehreren Kameras aufzeichnen und die Aufnahmen im Rahmen dieser Untersuchung veröffentlichen. Die Daten zeigen, dass ich bereits nach den ersten vier gemeinsamen Probentagen von den Teilnehmenden als Teil der Produktion behandelt wurde und die Vorstellung des „beobachtenden Wissenschaftlers“ immer weiter verblasste, bis selbst die Anwesenheit der Kameras als selbstverständlicher Teil des Projekts verstanden wurde.

21 Im Folgenden wird mit Probe die räumlich-zeitliche Herstellung von Situationen zum Zweck der Erarbeitung eines Stücks in der Institution Theater verstanden. Mit Probieren werden körperlich-kreative Prozesse der Künstler*innen (z. B. Modulationen, Variationen und Improvisationen einer Textvorlage) innerhalb von Proben bezeichnet. In einer Probe kann demnach unter anderem probiert, diskutiert oder geplant werden. Die einzelnen Proben werden je nach Schwerpunkt der Theaterschaffenden anders bezeichnet, z. B. unterscheidet sich eine Leseprobe, in der primär ein Text gemeinsam gelesen wird, von einer szenischen Probe, in der Text performativ umgesetzt wird (vgl. Umathum 2011; Zanetti 2011).

22 Um den Status Schutzraum auch nach Abschluss der Aufnahmen zu erhalten, wird auf alle Beteiligten nur durch ihre institutionelle Rolle im Setting Theaterprobe referiert. Hinweise auf den Aufnahmeort und Inhalte des Stücks werden nur eingeführt, wenn dies dem Verständnis der Rekonstruktion der Probenarbeiten dienlich ist. Alle Beteiligten willigten vor Aufnahmebeginn schriftlich ein, audiovisuell aufgezeichnet zu werden und waren mit der Veröffentlichung der Ergebnisse in anonymisierter Form einverstanden. Zur forschungsethischen Reflexion der Datenerhebung siehe Krug \& Heuser (2018). 
Die Proben fanden im Frühjahr 2016 an einem professionellen deutschen Stadttheater statt. Neben dem Regisseur, der Regieassistentin, einer Schauspielerin und einem Schauspieler waren an vielen Probentagen auch die Ausstatterin, der Dramaturg, der Inspizient, zwei Hospitant`innen und insgesamt drei Praktikant*innen anwesend. Später kamen noch ein Ton- und ein Lichttechniker sowie zwei Kinderdarsteller hinzu. Einzig der Dramaturg, die Regieassistentin und die Techniker waren vorher schon Teil des Theaterhauses. Alle anderen Beteiligten kamen als sogenannte Gäste für diese Produktion hinzu und arbeiteten zum ersten Mal gemeinsam zusammen, um ein Stück zu erarbeiten, in dem es um chirurgische Eingriffe bei Kindern gehen sollte. Dabei war geplant, nicht die traditionelle Form des „abbildenden Theaters“ (vgl. Perry 2010: 13) zu bedienen, sondern die Inszenierung als Stückentwicklung (bzw. devising theatre im englischen Sprachraum) zu gestalten. Unter diesen Begriff werden ,jene Theaterformen gefasst, die nicht ausgehend von einem dramatischen Text und dessen vorgegebener Handlungsstruktur arbeiten, sondern im Proben - im konzeptionellen Entwerfen und improvisierenden Hervorbringen - die Dramaturgie der Inszenierung entwerfen“ (Matzke 2011: 144). Dabei entstehen Theaterstücke aus dem gemeinsamen Zusammentragen von öffentlich zugänglichen oder selbstgeschriebenen Texten, Musikstücken oder Videos, die in Improvisationen eingesetzt und durch kritisches Hinterfragen zu dramatischen Texten und Spielszenen verdichtet werden. Während bei einigen Stückentwicklungen bewusst auf den Regieposten verzichtet wird (Magnat 2005: 79), findet sich in der begleiteten Stückentwicklung eine klassische Rollen- und Aufgabenverteilung der Beteiligten. Gemein haben die aufgezeichneten Proben mit den in der theaterwissenschaftlichen Literatur beschriebenen Stückentwicklungen (deLahunta 2002; Perry 2010) vor allem die gemeinsame Arbeit am Text, bei der von den Regieführenden, über die Schauspieler*innen bis hin zu Hospitanten ${ }^{\star}$ innen jede ${ }^{\star}$ r Ideen miteinbringen kann, was den Probenprozess maßgeblich mitprägt und gleichzeitig für einen „notwendigen Grad an Chaos“ (Magnat 2005: 75) sorgt. Damit lassen sich an einer Stückentwicklung Beteiligte auf die besondere Herausforderung ein, innerhalb von sechs Wochen ein zufriedenstellendes Stück zu entwickeln und dieses zum vorher festgesetzten Premierentag vor einem Publikum vorzuführen. Dies ist deutlich anspruchsvoller als bei einer Inszenierung, die bereits über eine dramatisierte und von einem Verlag redigierte Textvorlage verfügt. ${ }^{23}$ Gleichzeitig ist

23 Dies scheint zunächst einer der Gründe zu sein, weshalb die Teilnehmenden in der beobachteten Produktion so oft gleichzeitig verschiedene Aktivitäten bearbeiten. Tatsächlich sind aber komplexe Aktivitäten wie das SOUFFLIEREN oder das ANWEISENDE-VORMACHEN auch in Probenprozessen anderer Theaterformen bzw. das ABLEGEN VON KLEIDUNG WÄHREND EINES VoRSCHLAGS oder das TRINKEN WÄHREND EINES GESPRÄCHS auch in Alltagsinteraktionen vorstellbar. 
ein Scheitern der Stückentwicklung immer auch ein persönliches Scheitern aller an der kollektiven Arbeit Beteiligten. Auch wenn am Ende der ${ }^{\star}$ die Regisseur ${ }^{\star}$ in oder die Theatergruppe als Autor`in auf dem Textbuch im Verlag aufgeführt wird, bleibt es doch eine gemeinsame Leistung. Diese gemeinsame Leistung beginnt bereits in der allerersten Probe (die sog. Konzeptionsprobe ${ }^{24}$ ), in der die Beteiligten um einen Tisch herumsitzen, sich einander vorstellen und, geleitet durch den Regisseur, ihr persönliches Verhältnis zum im Stück verhandelten Stoff darlegen (Abb. B.1).

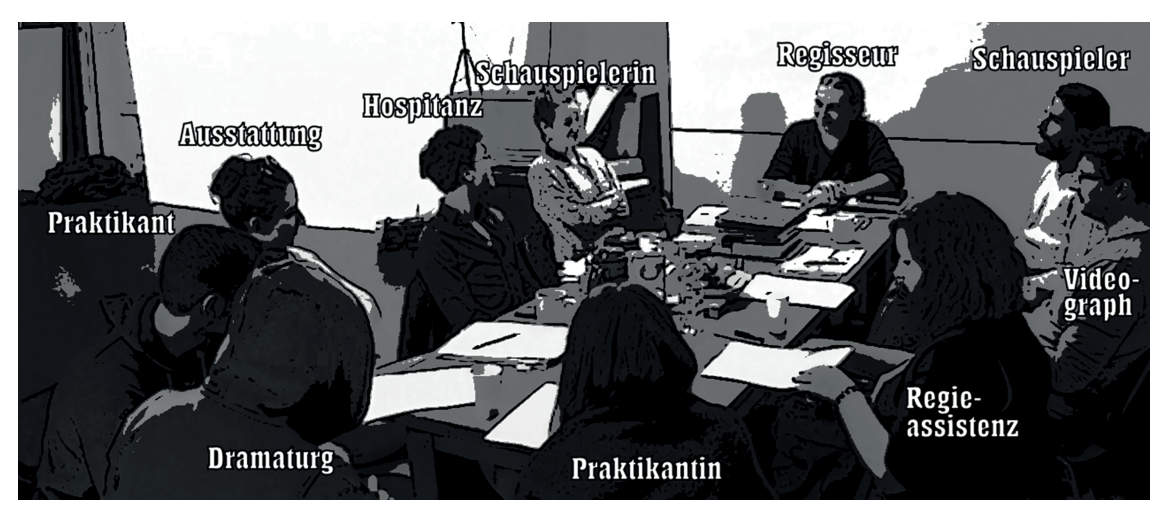

Abb. B.1: Die Beteiligten sitzen während der Konzeptionsprobe an einem großen Tisch.

Während dieser zum Teil sehr intimen Gespräche entwickeln sich Motive, die von den Beteiligten in späteren Probenphasen wieder aufgegriffen und im Theatertext verarbeitet werden. Dies geschieht in den Daten des Korpus nicht zufällig, sondern wird strikt arbeitsteilig von unterschiedlichen Beteiligten übernommen. In den ersten szenischen Proben improvisieren ${ }^{25}$ die Schauspielenden einzelne Spielsequenzen, während die Regieassistentin das Gesagte mitschreibt und Notizen zu Auf- und Abgängen oder benötigten Requisiten anfertigt (Abb. B.2).

Im Verlauf der Produktion kommt es an einigen Probentagen dazu, dass die Regieassistentin diese Mitschriften nach Probenschluss (oft gegen 22 Uhr) abtippt und sie dem Regisseur schickt. Dieser fertigt daraus eine Probenvorlage an, die er noch in derselben Nacht an die Schauspielerin und den Schauspieler der Produktion sendet, damit diese den Text als Grundlage für die Probe am nächsten Morgen verwenden können. Während mit Teilen dieses Textes dann wieder

24 Ein Überblick über verschiedene Probenarten findet sich bei Brauneck (2001).

25 Die Improvisation ist keinesfalls nur eine Technik der Stückentwicklung, sondern selbstverständlicher Teil von Probenprozessen traditionellerer Theaterformen (vgl. Eikels 2011). 
improvisiert werden kann, bleiben andere Passagen unangetastet. Damit verdichten sich die Improvisationen stetig zu einer festeren Text- und Darstellungsform. Gleichzeitig werden die so gebauten Szenen vor und nach den szenischen Proben von den Beteiligten am Tisch reflektiert, diskutiert und bei Bedarf redigiert.

Insgesamt umfasst das Korpus 800 Stunden Videomaterial des insgesamt 200-stündigen Probenprozesses von der Konzeptionsprobe bis zur Generalprobe. Um den oft wechselnden Konfigurationen aus Tisch- und Bühneninteraktionen, die häufig den gesamten Raum ausnutzen, gerecht zu werden, kam ein komplexes Setup an audiovisuellen Aufzeichnungsinstrumenten zum Einsatz.

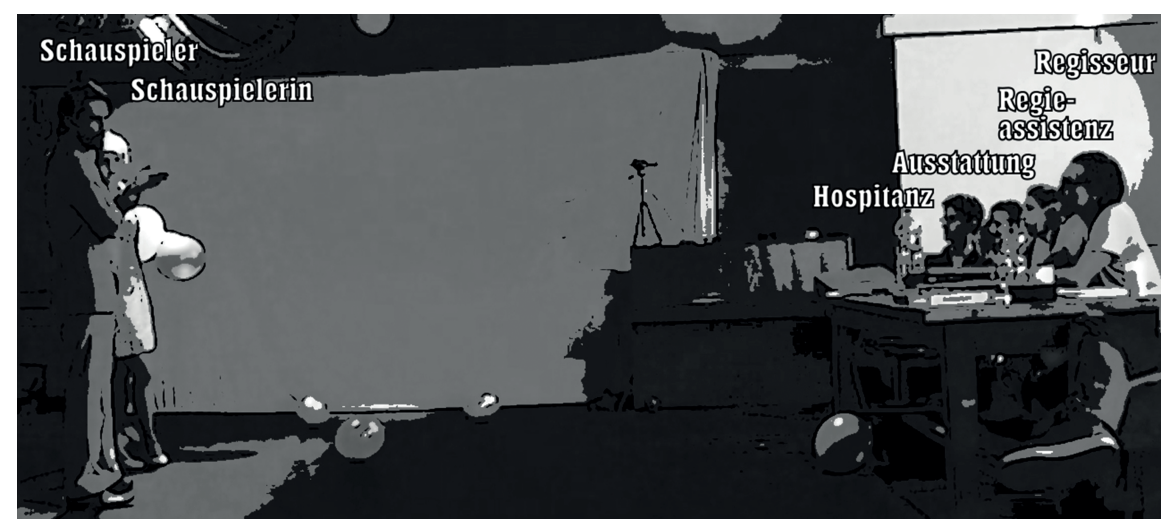

Abb. B.2: Während des Probierens agieren die Schauspielenden vor dem Regietisch.

Hinter dem Regietisch war eine Canon XA10 (1080p/25fps) aufgestellt, die über einen großen Dynamikumfang verfügt und damit sowohl überstrahlendes Scheinwerferlicht auf der Bühne als auch Low-Light-Situationen auf dem Regietisch zufriedenstellend abbilden kann. Der Nachtsichtmodus der Canon XA10 kam besonders ab dem Wechsel zur Spielstätte zum Einsatz, wenn unter realen Lichtbedingungen einer Aufführung geprobt wurde. Die XA10 verfügt über zwei SD-Karten-Slots auf die nonstop aufgezeichnet werden kann. Damit waren bis zu 8 Stunden Videoaufzeichnung möglich, ohne die Kamera anfassen und die Beteiligten so an die Aufnahmesituation erinnern zu müssen. In den meisten Fällen liefen die Kameras bereits, bevor die Theaterschaffenden die Räume betraten. Verbunden mit der XA10 war das Bluetooth-betriebene Mikrophon Canon WM-V1, das aufgrund seiner unauffälligen Form und Größe sowie der Audioqualität auf dem Regietisch positioniert wurde. Der Regietisch selbst wurde von einer Canon HF100 (1080p/25fps) aufgezeichnet, da diese Kamera besonders im Zwielicht scharfe Bilder produzieren kann. Neben der Canon HF100 war ein Zoom H4n 
Audiorecorder positioniert, der auf die Bühne ausgerichtet war. Ferner kamen ab der dritten Probe noch zwei Panasonic HC-X900M (1080p/25fps) zum Einsatz, die jeweils in einer der gegenüberliegenden Ecken der Probebühne bzw. Spielstätte positioniert waren. Durch diese Aufteilung der technischen Geräte konnte sichergestellt werden, dass unabhängig von der Position im Raum alle Beteiligten stets von mindestens einer Kamera audiovisuell erfasst wurden (Abb. B.3).

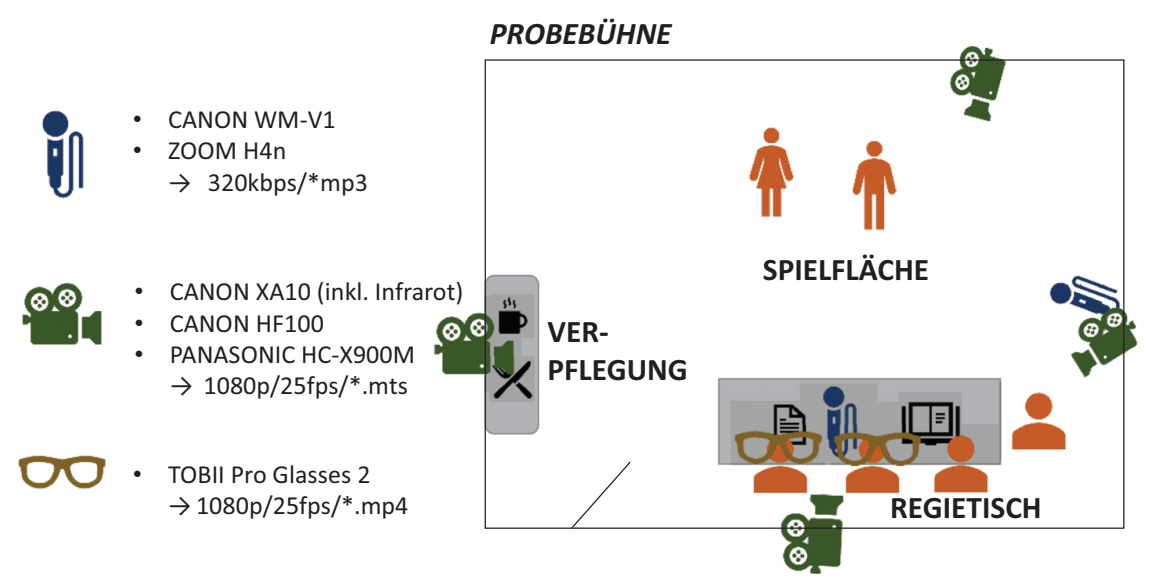

Abb. B.3: Aufnahme-Setup der Probebühne.

Zusätzlich zu diesem Multi-Kamera-Setup kamen in ausgewählten Proben (Einzelprobe und szenische Probe auf der Probebühne sowie erste Probe auf der Spielstätte und letzte Probe vor der Generalprobe) zwei mobile Eye-Tracking-Brillen (Tobii Pro Glasses 2) zum Einsatz. Dabei handelt es sich um technische Geräte in Brillenform, die Infrarotsignale an die Netzhaut des Trägers aussenden und aus der Reflexion die Position der Pupille errechnen. Dieser Wert wird mit einer Blickfeldkamera (1080p/25fps) kombiniert, die über dem Steg angebracht ist. Mithilfe der Software Tobii Pro Lab ergibt sich daraus ein Videobild, das die Blickbewegungen des Trägers inklusive Fixationen und Sakkaden anzeigen kann. Die Brillen werden mit einem Sicherheitsband am Hinterkopf der Träger fixiert und legen die Daten auf einer Aufnahmeeinheit ab, die per HDMI-Kabel mit der Brille verbunden ist. Beide Brillen zusammen zeichneten etwa 10 Stunden Material auf und wurden abwechselnd vom Regisseur, dem Schauspieler, der Regieassistentin und der Ausstatterin getragen. Zum Zeitpunkt der Aufzeichnung betrug die Akkulaufzeit der Brillen ca. 140 Minuten, die allerdings aufgrund der unangenehmen Tragweise und Bewegungseinschränkung nur von der Regieassistentin ausgereizt wurde. Mithilfe der Eye-Tracking-Technologie sind Einblicke in mensch- 
liche Interaktionsprozesse möglich, die Forschende durch Videoaufzeichnungen allein nicht erhalten könnten. Wie die vorliegenden Daten zeigen, stützen sich die Interagierenden bei der Koordination multipler Aktivitäten besonders häufig auf die Ressource Blick, weshalb den Eye-Tracking-Daten in Kapitel 7 eine besondere Beachtung zukommt. Der Umgang mit und die Interpretation dieser speziellen Daten wird an den jeweils relevanten Stellen vertieft.

Die folgende Grafik (Abb. B.4) zeigt einen Überblick über die einzelnen Proben im Korpus. Darin wird deutlich, dass die Form des freien Gesprächs, bei dem sich eher assoziativ über das Thema des Stücks unterhalten wird, vergleichsweise früh einer fokussierten Arbeit am Text weicht, der dann szenisch probiert wird. Diese Aufteilung der einzelnen Arbeitsphasen wird besonders in den ersten vier Proben deutlich. Erlebnisse, Erfahrungen und Meinungen aus der ersten Konzeptionsprobe werden bereits am zweiten Probentag als Textfragmente festgehalten und am dritten Tag zum ersten Mal auf der Probebühne ausprobiert. Die vierte Probe beinhaltet vor allem die Überarbeitung und Neuausrichtung des probierten Texts. Ab der fünften Probe bis zur letzten Möglichkeit in der Generalprobe (der letzten Probe vor der Premiere) wird der Text immer wieder überarbeitet, mit dem Wechsel von der Probebühne zur Spielstätte in der 20. Probe neu probiert und ab der 22. Probe auch mit Ton-, Licht- und Videotechnik abgestimmt. Um eine Vergleichbarkeit der analysierten Sequenzen zu gewährleisten, folgt die Datenauswahl der dieser Studie zugrundeliegenden Fallkollektionen dieser Aufteilung. Im Fokus stehen szenische Proben, innerhalb derer die Theaterschaffenden das Stück im engeren Sinne entwickeln. Ausgeschlossen werden damit die Probentypen der Konzeptions- und Leseproben sowie technische, Ausstattungs- oder Hauptproben. Innerhalb der szenischen Proben liegt der Schwerpunkt auf solchen Situationen, in denen die Beteiligten im Rahmen von Gesprächen sich über das Stück austauschen (Kapitel 5) oder für die Dauer von szenischer Arbeit am Stück selbst probieren (Kapitel 6 \& 7).

Mithilfe dieser Setzung werden vergleichbare Fallkollektionen möglich, in denen Interagierende simultan relevante Aktivitäten koordinieren. Probenaktivitäten des Schwerpunkts Gespräch umfassen dabei vor allem theaterunspezifische Aktivitäten, die auch in Alltagsinteraktionen beobachtbar sind wie das SMARTPHONE NUTZEN, die TASCHE AUSPACKEN, die JACKE ABLEGEN oder BEgRÜSSEN, BERICHTEN sowie Vorschlagen (Kapitel 5). Theaterspezifisch sind hingegen die Multiaktivitäten wie eine TANZENDE-ANWEISUNG oder eine TANZENDE-BITTE (Kapitel 6) sowie die Soufflage (Kapitel 7), mit denen die Theaterschaffenden bestimmte Aspekte des Projekts Entwickeln eines Theaterstücks bearbeiten. Mithilfe der Aufteilung nach Phasen im Probenprozess kann bei der Auswahl der Einzelsequenzen zweierlei sichergestellt werden. Erstens, dass jedem Fall mindestens ein vergleichbarer Komplementärfall in Bezug auf die Aktivitätskategorien (Einzel-/Ensembleaktivitä- 
ten) gegenübergestellt wird: Wenn in Transkript 1 der Abbruch einer Einzelaktivität zugunsten einer Ensembleaktivität betrachtet wird, kann dies mit dem Abbruch einer Ensembleaktivität zugunsten einer Einzelaktivität ohne (Transkript 2) und mit Account (Transkript 3) verglichen werden. Da diese systematische Auswahl über die Analysekapitel 5-7 hinweg konstant gehalten wird, lassen sich die Fallkollektionen sowohl innerhalb der Kapitel (mit den jeweiligen Koordinierungsschwerpunkten) als auch kapitelübergreifend miteinander vergleichen.

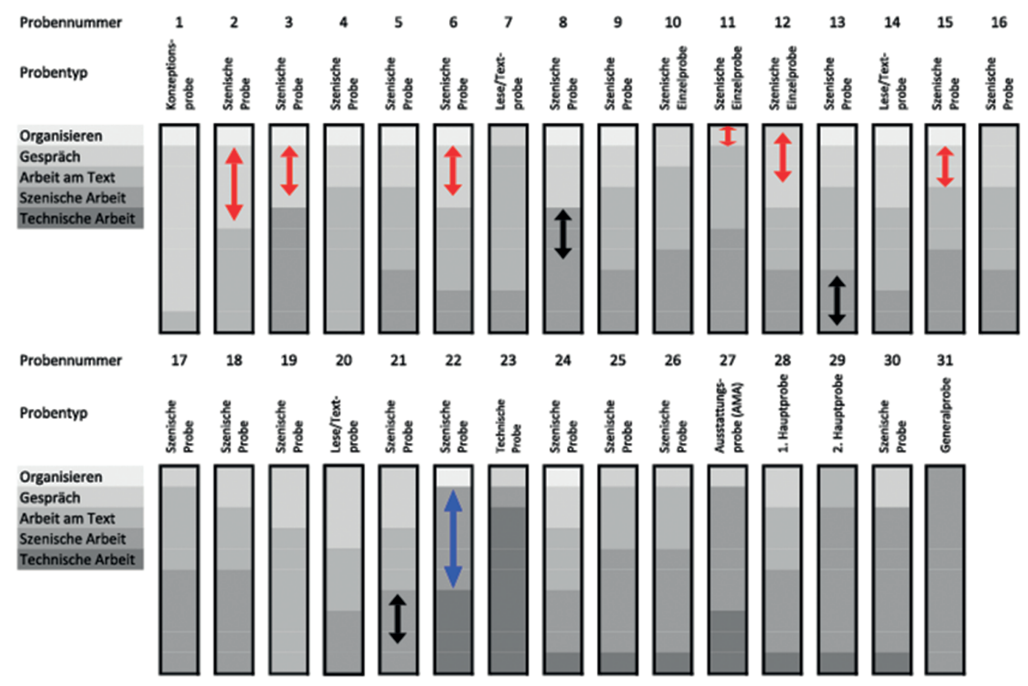

Roter Pfeil: Ausschnitt, der in Kapitel 5 „Strukturelle (In)Kompatibilität der Beteiligungsweisen” besprochen wird Blauer Pfeil: Ausschnitt, der in Kapitel 6 „Strukturell kompatible Ensembleaktivitäten” besprochen wird Schwarzer Pfeil: Ausschnitt, der in Kapitel 7 “Strukturell inkompatible Einzelaktivitäten” besprochen wird

Abb. B.4: Übersicht des Probenkorpus, Näherungswerte auf Grundlage des ethnographischen Feldtagebuchs der teilnehmenden Beobachtung mit Verortung der betrachteten Sequenzen in den Analysekapiteln 5-7.

Zweitens sorgt die Fokussierung auf unterschiedliche Phasen im Probenprozess dafür, dass die eben beschriebene Vergleichbarkeit sichergestellt ist: Es wird bei der Auswahl der Einzelsequenzen stets darauf geachtet, strukturell ähnliche Aktivitäten miteinander zu vergleichen. Das sind in Kapitel 5 gesprächsförmige Ensembleaktivitäten (BERICHTEN, ERKLÄREN, VoRSCHLAGEN, usw.) und Einzelaktivitäten mit primär haptischer Beteiligungsweise (RUCKSACK AUSPACKEN, JACKE AUSZIEHEN, AM SMARTPHONE TIPPEN, usw.). Die Fallanalysen in Kapitel 6 fokussieren im Unterschied dazu koordinative Verbindungen von TANZ-Ensembleaktivitäten mit primär kinästhetischen Beteiligungsweisen mit gesprächsförmigen Ensembleaktivitäten wie ANWEISEN, BITTEN ODER VoRSCHLAGEN. In Kapitel 7 schließlich 
werden solche Fälle analysiert, in denen Einzelaktivitäten mit primär visueller Beteiligungsweise (LESEN, SCHREIBEN, MONITOREN) mit der eher gesprächsförmigen Ensembleaktivität SZENISCHES SPIEL koordiniert werden.

Wie in Abb. B.4 mithilfe der farbigen Pfeile angezeigt ist, werden in den einzelnen Analysekapiteln Sequenzen unterschiedlich vieler Probentage untersucht. Während in Kapitel 5 eine breitere Auswahl von Einzelsequenzen aus der eher gesprächsintensiveren ersten Hälfte des Probenprozesses zugrunde liegt, fokussieren Kapitel 6 und 7 speziellere Aspekte der Proben. Dies zeigt sich für Kapitel 6 darin, dass dort die Arbeit einer Tanzerarbeitung als Unterprojekt innerhalb des übergeordneten Projekts Theaterstück fokussiert wird. Die Theaterschaffenden beginnen und beenden dieses Projekt Tanzerarbeitung in der 22. Probe, wodurch der Erarbeitungsfortschritt deutlich einfacher als für das übergeordnete Projekt nachgehalten werden kann. Da in Kapitel 7 die Koordinationsprozesse von Einzelaktivitäten wie LESEN, SCHREIBEN SOwie MONITOREN analysiert werden sollen und dabei vor allem visuelle Beteiligungsweisen zum Einsatz kommen, bietet sich hier ein analytischer Zugriff über das Messinstrument des mobilen Eye-Trackings an. Um die theatrale Arbeit durch das im Vergleich zu reiner Videographie eher intrusive Eye-Tracking nicht zu stören (vgl. Krug \& Heuser 2018), kommt dieses Messinstrument nur an vier gleichmäßig über den Gesamtprozess verteilten Probentagen (Tag 8, Tag 13, Tag 21 und Tag 30) zum Einsatz. Am Tag vor der Generalprobe (Tag 30) tritt die fokussierte Multiaktivität SouffLAGE nicht auf, sodass sich die Auswahl der Einzelsequenzen auf die übrigen drei Probentage fokussiert.

Da es sich bei Proben, wie eingangs erläutert, um Schutzräume handelt, werden alle Daten des Korpus dergestalt anonymisiert, dass keine Rückschlüsse auf Einzelpersonen oder Institutionen möglich sind. Neben den audiovisuellen Daten beinhaltet das Korpus ebenfalls ausführliche ethnographische Notizen in Form eines Feldtagebuchs, Fotographien der Räumlichkeiten, Kopien der angefertigten Dokumente (z. B. Mitschriften der Regieassistentin), Interviews mit den Beteiligten während und nach den Probenarbeiten, analysegeleitete Expertengespräche mit externen professionellen Theaterschaffenden, Kritiken und öffentliche Kommentare zum Stück sowie alle Versionen des Textbuchs. Diese Daten werden gleichrangig neben den Videodaten behandelt und bei Bedarf in die Analysen mit einbezogen. Damit stützt sich der Ansatz dieser Untersuchung auf den Vorschlag von Deppermann (2000: 103-104), „Ethnographie als Ergänzung zur Konversationsanalyse“ zu nutzen, um „die Kultur eines Feldes in ihrer ,Gesamtheit‘, was heißt: in ihren wesentlichen Strukturen, Prozessen und deren Bezügen zueinander in den Blick zu bekommen.“ Das bedeutet, dass aufgrund eines streng datengeleiteten Vorgehens nur Aussagen über die belegbaren interaktiven Praktiken der Beteiligten möglich sind. Ebenfalls können so nur Aussagen über die Proben dieser Stückentwicklung gemacht werden, da es keine festgeschriebe- 
nen Probenregeln am Theater gibt (Matzke 2012: 27). Dennoch ist das Ziel dieser Untersuchung verallgemeinerbare Ergebnisse $\mathrm{zu}$ Koordinationspraktiken bei multiplen Aktivitäten zu liefern, die auch in anderen, nicht-institutionellen Interaktionen beobachtbar sind.

\subsection{Exkurs: Proben in der Institution Theater}

Theaterproben sind Arbeitsphasen in der Institution Theater. Als Teil eines institutionellen Arbeitsbereichs unterscheiden sie sich von häuslichen und öffentlichen Situationen vor allem im Grad ihrer Planbarkeit, der räumlichen und zeitlichen Gebundenheit sowie der spezifischen Teilnehmendenrahmung. Die Untersuchung institutioneller Arbeitsbereiche hat im Zuge der sogenannten Workplace studies eine lange Tradition in der Soziologie und Linguistik. Empirische Arbeiten zu Gerichtsprozessen (Atkinson \& Drew 1979), medizinischen Untersuchungen (Heath 1986), Call-Centern (Mondada 2008) oder Flugverkehrsüberwachungen (Arminen, Koskela \& Palukka 2014) ermöglichen neue Perspektiven auf soziale Interaktionen in komplexen Umgebungen. Die vorliegende Untersuchung der Theaterproben reiht sich in die Tradition der Workplace studies ein und beschreibt Probenprozesse als Zusammenspiel institutionell verfestigter Aktivitäten, die von den Teilnehmenden gleichzeitig bearbeitet werden.

Die institutionelle Verankerung der Theaterprobe geschieht theatergeschichtlich erst vergleichsweise spät. Bis in das 18. Jahrhundert hinein sind Proben nicht nur unüblich und gelten als Zeitverschwendung, sondern werden auch ,als Strafe verstanden zur Disziplinierungsmaßnahme für die Schauspieler“ (Matzke 2012: 21). Den Probenprozess als gemeinsame Vorbereitung und als Arbeit an einem Stück zu sehen, beginnt erst nach der Einführung der Weimarer Theatergesetze 1793 durch Goethe (1960 [1793]). Dieses Schriftstück regelt das selbstständige Erscheinen der Schauspieler zu den Proben, verbietet Trunkenheit bei Proben und setzt die Höhe der Strafen bei Regelverstößen fest. Goethe wird damit zum Pionier des probenintensiven Regietheaters (Matzke 2012: 130): „Wenn Goethe in einer Handschrift von 1794 die Proben zum Stück Die Geschwister auf dem Lande auf acht Tage verteilt, unterschieden in Leseprobe und Hauptprobe, dann ist das viel im Verhältnis zu den üblichen Probenzeiten Ende des 18. Jahrhunderts.“ Mit den Theatergesetzen beginnen die Proben immer untrennbarer mit der Institution Theater zu verwachsen. Erste Anzeichen dafür liefern die Ausformungen der Probe zum Arbeitsort mit festgelegten Positionen. In der Mitte des Probenraumes sitzt die Regie, an ihrer Seite die Assistenz und auf der Probebühne befinden sich nur diejenigen Schauspieler, deren Szene gerade geprobt wird - eine Aufteilung, an der sich bis heute nichts geändert hat. Zudem wird ver- 
mehrt das Publikum von den Proben ausgeschlossen und der Proberaum als Schutzraum für die Kunstschaffenden etabliert (Matzke 2012: 256).

Im 19. Jahrhundert wird die Ausdifferenzierung der Proben weiter vorangetrieben. Probenprozesse bestehen nun aus mindestens drei Probenarten: dem gemeinsamen Lesen des Stücks (Leseprobe), dem Durchplanen einzelner Szenen (Setzprobe) sowie der Aufführungssimulation (Haupt-/Generalprobe) (vgl. Roselt 2011). Durch die anspruchsvolleren Prozesse wird eine Arbeitsteilung festgelegt. Neben Schauspieler^innen, der Regie und der Assistenz sind nun auch Theatermeister*innen, Requisiteur*innen und Soufflierende bei der Leseprobe anwesend. Allerdings bleibt die herausgehobene Stellung der Regieführenden unangefochten. So erörtern Regisseur`innen und Schauspieler`innen auf der Setzprobe zwar die Szenen, die Darstellungen der Schauspieler`innen werden aber nur markiert und die Auf- und Abgänge in ein „Setz-Scenarium“ geschrieben, dem Vorläufer des heutigen Regiebuchs. Da der Regisseur alle Aktionen auf der Bühne festlegt, sind keine Korrekturvorschläge oder Anmerkungen seitens der Schauspielenden notwendig (vgl. Roselt 2011: 24-25). Auch bleibt es zu Beginn des 19. Jahrhunderts bei vier Proben pro Stück, um die Schauspieler^innen nicht unnötig auszulaugen. Häufig findet daher erst bei der Premiere vor Publikum der erste Durchlauf statt. Proben im 19. Jahrhundert sind daher vor allem als Vermittlungsarbeit eines Textes durch die Regie für die Schauspieler`innen zu verstehen (vgl. Blum, Herloßsohn \& Marggraf 1846: 125).

Dieses Vorgehen verändert sich grundlegend unter Berthold Brecht im 20. Jahrhundert. Bei ihm sind 160 Proben keine Seltenheit, dem russischen Regisseur und Theatertheoretiker Konstantin Sergejewitsch Stanislawski (1987) werden sogar 200 Proben nachgesagt (vgl. Matzke 2012: 240). Geprobt wird über mehrere Monate hinweg unter der Anwesenheit von Techniker^innen, Bühnenarbeiter^innen,

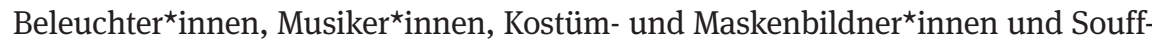
leur`eusen. Die expandierten Probenphasen tragen dem neuen Selbstverständnis des Theaters Rechnung, bei dem es nicht mehr um die Reproduktion einer geschriebenen Vorlage geht, sondern um die Auseinandersetzung mit dem Stoff. Infolgedessen entsteht das Probieren in Theaterproben aus Improvisationen mit Text im szenischen Spiel. Damit wird die Alleinherrschaft der Regieführenden aufgelöst, wie eine Anekdote aus dem Probenprozess von Brecht illustriert: „Brecht rief seine Anweisungen meist von seinem Stuhl aus, der gute zehn Meter von der Bühne entfernt stand, den Schauspielern zu. Die immer sehr kurzen Beratungen am Regietisch wurden protokolliert. Gab es zwei Auffassungen, wurden beide probiert“ (Matzke 2012: 178). Zwar hat die Regie nach wie vor als künstlerische`r Leiter^in die Entscheidungsgewalt darüber, welche der Varianten in die Inszenierung aufgenommen werden soll, dennoch weichen im 20. Jahrhundert die Anweisungsproben einem kollektiven Ansatz des Probierens, bei der die Beteiligten gemeinsam an der 
in den Proben entworfenen Inszenierung arbeiten. Die Stückentwicklung als Theaterform des 21. Jahrhunderts setzt diese Entwicklung konsequent fort. Allerdings geht die Quantität der Proben aus ökonomischen Gründen wieder zurück. Für die dieser Untersuchung zugrundeliegende Produktion waren 31 Proben inklusive der Generalprobe angesetzt.

Theaterproben in professionellen Häusern sind nach vier Jahrhunderten Entwicklung heute selbstverständlicher Teil der Institution und bilden den Kern des Theaterbetriebs, um den alle anderen Arbeiten am Theater herum geplant werden. Die Institutionalisierung der Proben wird besonders deutlich durch die Arbeitsteilung betrieblich vorgegebener Rollen und Funktionen. Neben den Mitarbeiter*innen des Künstlerischen Betriebsbüros (KBB), die beispielsweise dafür sorgen, dass die Probebühnen den Probenden überhaupt zur Verfügung stehen, tragen die Kantinen- und Reinigungskräfte mit ihren Aktivitäten ebenfalls dazu bei, dass die Institution Theater funktionieren und Proben (und damit Aufführungen) möglich sein können. Während der Probenzeiten von 11 Uhr bis 22 Uhr, wie sie in der für diese Untersuchung begleiteten Produktion keine Seltenheit waren, konzentrieren sich die an einer Probe Beteiligten (Regisseur`in, Assistenz, Schauspieler`in, Dramaturg ${ }^{\star}$ in, Techniker*innen) ausschließlich auf ihre Arbeit: das Proben. Vor und nach den Proben werden die Reinigungskräfte und Kantinenmitarbeiter*innen aktiv, die die Spielflächen sauber halten und in den Pausenzeiten zwischen 15 Uhr und 19 Uhr Mahlzeiten ermöglichen. Der gesamte betriebliche Ablauf im Theater ist dahingehend optimiert und arbeitsteilig organisiert, dass die Kernarbeit am Theater, das Schaffen von Theaterstücken, möglich sein kann. Damit trägt die Institution dem immensen Druck Rechnung, der auf den Theaterschaffenden lastet, ein Theaterstück in nur sechs Wochen zu inszenieren und im Fall einer Stückentwicklung zusätzlich erst noch von Grund auf zu schreiben. Da die Spielzeit des jeweiligen Hauses bereits im Vorfeld festgelegt und angekündigt ist, steht auch der Premierentermin schon weit im Voraus fest und kann aus ökonomischen Gründen in der Regel nicht verschoben werden. Für alles, was innerhalb der Probenzeit nicht fertig geworden ist, „haften“ die Schauspieler`innen und die Regieführenden bei der Aufführung vor dem Publikum mit ihren Namen und Gesichtern.

Die aus dieser zeitlichen Beschränkung resultierende klare Zielorientierung charakterisiert den Großteil aller Interaktionen innerhalb von Theaterproben. Um mit der knappen Zeit möglichst effizient umzugehen, wird eine strenge Arbeitsteilung eingehalten. Trotz des vergleichsweise offenen Formats der Stückentwicklung steht in der begleiteten Produktion der Regisseur als künstlerischer Leiter an der Spitze der Probenhierarchie. Danach kommen Schauspielerin und Schauspieler, die zusammen mit dem Regisseur das Kernteam der Inszenierung bilden und deren Anfragen mit höherer Priorität bearbeitet werden. Darauf folgen 
die für den Probenablauf zuständige Regieassistenten, der in Bezug auf den Text beratende Dramaturg und die für Bühnen- und Kostümbild verantwortliche Ausstatterin sowie die ausführenden Techniker^innen. Hospitanten`innen sind der Regieassistenz unterstellt und in den Daten größtenteils damit beschäftigt, Kaffee zu kochen, Obst zu schneiden oder kleinere von der Regieassistenz delegierte Aufgaben, etwa das Drucken der neuen Stückfassung, zu übernehmen. Anders als in anderen Bereichen des Theaterbetriebs, in denen aktiv mitgearbeitet werden kann, wird von Praktikanten^innen in den Probenzeiten vor allem erwartet, pünktlich zu erscheinen und still zuzuhören. Diese Hierarchie lässt das Probieren der Szenen durch die Schauspieler`innen und den Regisseur in den Proben ins Zentrum rücken. Je weniger eine institutionelle Rolle in den Theaterproben mit dem Probieren zu tun hat, desto peripherer wird sie. Deutlich wird das vor allem in der Teilnehmendenrahmung, die bereits während der gemeinsamen Gespräche in der Konzeptionsprobe anhand der asymmetrischen Verteilung des Redeanteils deutlich wird: Regisseur und Schauspieler*in beanspruchen einen Großteil des Rederechts für sich; die anderen Beteiligten sprechen meist nur nach Aufforderung. Diese Beschränkungen, die unter anderem bestimmen, was von den Teilnehmenden als erlaubte Interaktionsbeiträge in der jeweiligen Situation behandelt wird, ist nach der Zielorientierung und den spezifischen Teilnehmendenrahmungen ein Merkmal institutioneller Interaktion (vgl. Drew \& Heritage 1992: 22). Dieses Verhalten beruht auf dem geteilten Wissen der Beteiligten über die Institution Theater, das sie im Studium, Ausbildung und im Laufe ihrer Arbeit an Theatern erworben haben. Dazu gehört nicht nur der selbstverständliche Gebrauch der institutionellen Fachsprache, dem sogenannten Theaterjargon ${ }^{26}$ (Rohr 1952; Mehlin 1969; Mundi 2005), sondern auch das Wissen über die Organisation in Phasen des Arbeitsprozesses mit immer wiederkehrender Abfolge von Probenarten. Konzeptionsproben, Leseproben, Spielproben, technische Proben, Ausstattungsproben, Haupt- und Generalproben finden sich in allen Theaternproduktionen wieder und bringen jeweils ein spezifisches Set an Anforderungen für die Beteiligten mit sich, auf das Theaterschaffende aufgrund ihrer Professionalität routiniert reagieren können.

Der Fokus auf die Kernaktivität des Probierens hat aufgrund der starken zeitlichen Limitierung Auswirkungen auf die Arbeitszeiten der Beteiligten. Alles, was nicht unbedingt während der Probenzeiten erledigt werden muss, wird ausgelagert. So lernen die Schauspieler`innen in ihrer Freizeit ${ }^{27}$ vor den Proben den Text

26 Im Folgenden wird solcher Theaterjargon nach einer kurzen Erklärung als kursive Formatierung markiert.

27 Der Begriff Freizeit ist im Rahmen des Theaters irreführend, wie Matzke (2012: 33f.) darstellt: „Der Künstler arbeitet zu jeder Zeit, und alles wird ihm zu Arbeit: an sich selbst, an seinen Ideen 
für die jeweilige Szene und sammeln im Fall von Stückentwicklungen zusätzlich Material für das Stück oder arbeiten an den Biographien ihrer Figuren. Im Fall des Regisseurs oder der Regieassistenz werden Probenpläne erstellt, Requisiten organisiert oder technische Wünsche an die jeweiligen Stellen im Theater weitergeleitet. In den Pausen der Proben diskutieren die Beteiligten entweder im Proberaum oder in der Kantine inszenatorische Fragen und auch nach Probenschluss ist die Arbeit an der Inszenierung nicht abgeschlossen. In geselliger Runde werden dann Aspekte besprochen, die während der Probenzeiten aufgrund der Dringlichkeit anderer Fragen hintenangestellt werden mussten. Im Fall der konkreten Stückentwicklung in den Daten wurde nach Probenschluss ebenfalls der Text für die Szene am nächsten Tag durch die Regieassistenz abgeschrieben und den Schauspielern zum Lernen weitergeleitet. Werden diese Tätigkeiten in Betracht gezogen, kann die Arbeit an einer Inszenierung nicht mit dem Ende der Probenzeiten gleichgesetzt werden. Die oftmals konzeptionellen und organisatorischen Arbeiten, die außerhalb der künstlerisch orientierten Probenzeiten stattfinden, tragen ebenfalls zum Gelingen der Produktion bei. Eine ethnographische Studie mit dem Ziel, eine Theaterproduktion vollständig beschreiben zu wollen, müsste demnach die Theaterschaffenden die vollen sechs Wochen begleiten und dürfte sich nicht nur auf die Proben konzentrieren. Bei einer Nicht-Stückentwicklung kommt zudem eine Phase der Vorproduktion hinzu, in der Konzepte geschrieben, Besetzungslisten angefertigt und erste Textfassungen erstellt werden. Die vorliegende Untersuchung hat indes ein anderes Ziel: diejenigen Verfahren zu identifizieren, mit denen die Theaterschaffenden innerhalb des zeitlichen (institutionell vorgegebene Probenzeiten mit eindeutigem Enddatum innerhalb routinierter Arbeitsphasen) und räumlichen (Proberaum innerhalb des Theaterhauses) institutionellen Rahmens mehrere gleichzeitig auftretende Aktivitäten interaktiv organisieren.

oder der konkreten Aufführung. Das Theatermachen hört für den Regisseur, den Schauspieler, die Kostümbildnerin weder am Abend noch mit der Premierenvorstellung auf.“ 